\title{
Krankheitsbedingte Fehlzeiten des Pflegepersonals eines Maximalversorgers - Analyse und Handlungsanweisungen
}

\author{
Johann Röper ${ }^{1}$ Steffen Fleßa' ${ }^{1}$ \\ Eingegangen: 5. Juni 2019 / Angenommen: 28. November 2019 / Online publiziert: 21. Februar 2020 \\ (c) Der/die Autor(en) 2020
}

\section{Zusammenfassung}

Hintergrund Krankheitsbedingte Fehlzeiten stellen im gesundheitsbetrieblichen Leistungserstellungsprozess Produktivitätsminderungen dar. Angesichts knapper Pflegefachkräfte, des demografischen Wandels und der Veränderung des Krankheitspanoramas sind die Kenntnis und das Management von Ausfällen in der betrieblichen Zielkonzeption zu verankern, um die Leistungsbereitschaft sicherzustellen. Für die systematische Einordnung möglicher Ausfallursachen und Ableitung von Maßnahmen zur Vermeidung von Produktivitätsverlusten wird ein auf die Krankenpflege ausgerichtetes Modell entwickelt und vorgestellt. Dieses soll die Analyse von Fehlzeitendaten der Pflegekräfte eines maximalversorgenden Krankenhauses strukturieren.

Ziel Ziele dieser Studie sind die Entwicklung eines Analysemodells für Fehlzeiten von Pflegepersonal in Krankenhäusern und dessen Anwendung auf Fehlzeitendaten eines maximalversorgenden Krankenhauses. Mithilfe des Modells sollen die Ursachen für Fehlzeiten systematisch erfasst, strukturiert und schließlich analysiert werden. Aus den Analyseergebnissen sollen schließlich Ansatzpunkte, wie sich Fehlzeiten betrieblich steuern lassen könnten, abgeleitet werden.

Methodik Zur Analyse von Fehlzeiten im Pflegeberuf wird ein systematischer Ordnungsrahmen entwickelt. Die Anwendung der Analysemethode erfolgt am Beispiel eines deutschen maximalversorgenden Krankenhauses, das Daten zur wissenschaftlichen Auswertung zur Verfügung stellte. Im Betrachtungszeitraum von 2 Jahren wurde ein Datensatz von 73.996 registrierten Ausfällen von rund 1500 Pflegekräften explorativ analysiert.

Ergebnisse Das Modell zur Analyse von Fehlzeiten beweist seine Anwendbarkeit für die Strukturierung und die systematische Erfassung von Ausfallursachen. Der erarbeitete Denkrahmen liefert damit sowohl einen wissenschaftlichen als auch praktischen Beitrag und kann in weiteren Fehlzeitenanalysen Anwendung finden. Als wichtiger Ansatzpunkt zur Reduktion der Fehlzeiten am Erfahrungsobjekt erweist sich die Homogenisierung der Personalstruktur, die an den Organisationeinheiten ansetzen sollte. Diese kann z. B. über den Altersdurchschnitt gesteuert werden, da den Ergebnissen zufolge ältere Pflegekräfte im Durchschnitt deutlich höhere krankheitsbedingte Fehlzeiten verursachen als junge. Männliche Pflegekräfte fehlten indes krankheitsbedingt öfter als weibliche. Darüber hinaus sollte anhand von Mitarbeitergesprächen regelmäßig der Kontakt zur Belegschaft gesucht werden, um motivationsbedingte Fehlzeiten zu verhindern und frühzeitig Kenntnis über etwaige Probleme zu erlangen.

Fazit Die erarbeitete Analysemethode ermöglicht die systematische Erfassung und Untersuchung von Fehlzeiten. Eine Reduktion der Fehlzeiten ist möglich. Sie erfordert die konsequente Umsetzung von Maßnahmen, die sich in der betrieblichen Zielkonzeption wiederfinden müssen.

Schlüsselwörter Betriebliches Gesundheitsmanagement · Fehlzeiten · Fehlzeitenmanagement · Krankenpflege · Leistungsbereitschaft $\cdot$ Personalmanagement $\cdot$ Produktivität

Johann Röper

jwa.roeper@gmx.de

Steffen Fleßa

steffen.flessa@uni-greifswald.de

1 Lehrstuhl für Allgemeine Betriebswirtschaftslehre und Gesundheitsmanagement, Universität Greifswald, Friedrich-Loeffler Str. 70, 17489 Greifswald, Deutschland 


\section{Absence due to illness of nursing personnel at a tertiary German hospital-Analysis and recommendations}

\section{Abstract}

Background Illness-related absences from work represent a reduction in productivity. Healthcare businesses are confronted with a scarce availability of nursing personnel, which is exacerbated by demographic change and a shift of the disease panorama. The management of absence times therefore requires a root cause analysis and should be integrated into an organization's strategy. A model to systematically analyze the possible causes of the recorded numbers of sick leave periods and the derivation of measures to avoid loss of productivity is presented. The aim was to fully and systematically grasp possible causes for absences and focusses both on scientific and practical usability in a tertiary hospital.

Objective The aim of this study was to develop a model to analyze the periods of absence of nursing personnel and to use it for data from a tertiary hospital in Germany. By using the model possible reasons for periods of absence were systematically documented, structured and finally analyzed. Based on the findings measures to reduce absences were developed to gain a better control of absence times.

Method A systematic approach to analyze times of absence in hospital nursing was developed based on the literature and specified on the profession of nursing. The approach was then used on the example of a German hospital. The analysis covers a time frame of 2 years during which 73,996 recorded absences by approximately 1500 nurses were investigated.

Results The model for analysis of absence periods confirmed its suitability for structuring and systematic documentation of causes of absences. To reduce absences the personnel structure of every organizational unit has to be adapted to achieve more balanced absence times. The average age on every unit can be used as a possible indicator as older nurses have higher absence times than younger nurses; however, male nurses on average caused higher sickness-related absence times than female nurses. Appraisal interviews should be used to gain better contact to nurses, to raise awareness of possible problems and to further avoid motivational absences.

Conclusion The presented analytical method can be used as a tool to systematically register and investigate illness-related absences. Their reduction is possible but requires the consistent implementation of measures, which have to be part of a hospitals target concept.

Keywords Rate of absence $\cdot$ Healthcare management $\cdot$ Human resources management $\cdot$ Nursing $\cdot$ Care $\cdot$ Motivation

\section{Hintergrund}

Die Erstellung von Gesundheitsdienstleistungen, die nach dem Uno-actu-Prinzip in Einheit von Raum, Ort und Zeit erfolgt, ist ein multidisziplinärer und hochintegrierter Prozess, bei dem die Arbeit des Pflegepersonals als elementarer Produktionsfaktor eine zentrale Leistungskomponente darstellt (Fleßa 2013). Nach ihrem Berufsethos ist es das Ziel der Krankenpflege, Pflegebedürftigkeit festzustellen, um bei Gesunden und Kranken aller Altersgruppen die notwendige Hilfe zu Erhaltung, Anpassung und Wiederherstellung von physischen, psychischen und sozialen Funktionen und Aktivitäten zu leisten (Deutscher Berufsverband für Pflegeberufe 1992). Krankenpflege kann auch als Beziehungsdienstleistung charakterisiert werden, die von inner- und zwischenmenschlichen Prozessen geprägt ist (Herrmannsdorfer 2000). Die Motivation zur Krankenpflege ist daher grundsätzlich anthropozentrisch (Duppel 2005). Häufig entstehen Krankheiten im Pflegeberuf durch psychosoziale und körperliche Belastungen, etwa durch körperliche Arbeit beim Bewegen von Patienten, oder durch den häufigen Kontakt zu schwer Kranken und Sterbenden (Badura et al. 2016; Glaser und Höge 2005). Fehlzeiten des Pflegepersonals, al- so eine Minderung der verfügbaren Arbeitsleistung, können die Effizienz und die Qualität der betrieblichen Leistungserstellung reduzieren. Eine schlechtere Pflegequalität trifft zum einen die Patienten und führt zum anderen zu steigenden Kosten, wenn beispielsweise die Verweildauer steigt oder zum Ausgleich zusätzliches Personal eingestellt werden muss. Zur Einhaltung des wirtschaftlichen Effizienzprinzips sind deshalb Maßnahmen wie das Halten oder Wiederherstellen des medizinischen Normalzustands (World Health Organisation 1948) des Pflegepersonals erforderlich. Krankheitsbedingte Fehlzeiten müssen also im Managementzyklus gesteuert werden und finden sich somit in der betrieblichen Zielkonzeption wieder (Fleßa 2012).

Zum Begriff Fehlzeiten liegt bislang keine allgemein anerkannte Definition vor (Brandenburg und Nieder 2009). In dieser Arbeit sollen Fehlzeiten als die Zeit verstanden werden, in der ein Arbeitnehmer das vorgesehene Arbeitspensum seiner Stelle aufgrund seiner Abwesenheit nicht leisten kann (Gabler Wirtschaftslexikon 2019a). Werden Fehlzeiten in Verbindung zum Gesundheitszustand gesehen, sind sie eine Kennzahl des Krankenstands und weisen auf „fehlende Gesundheit“ hin (Ulich und Wülser 2012). 


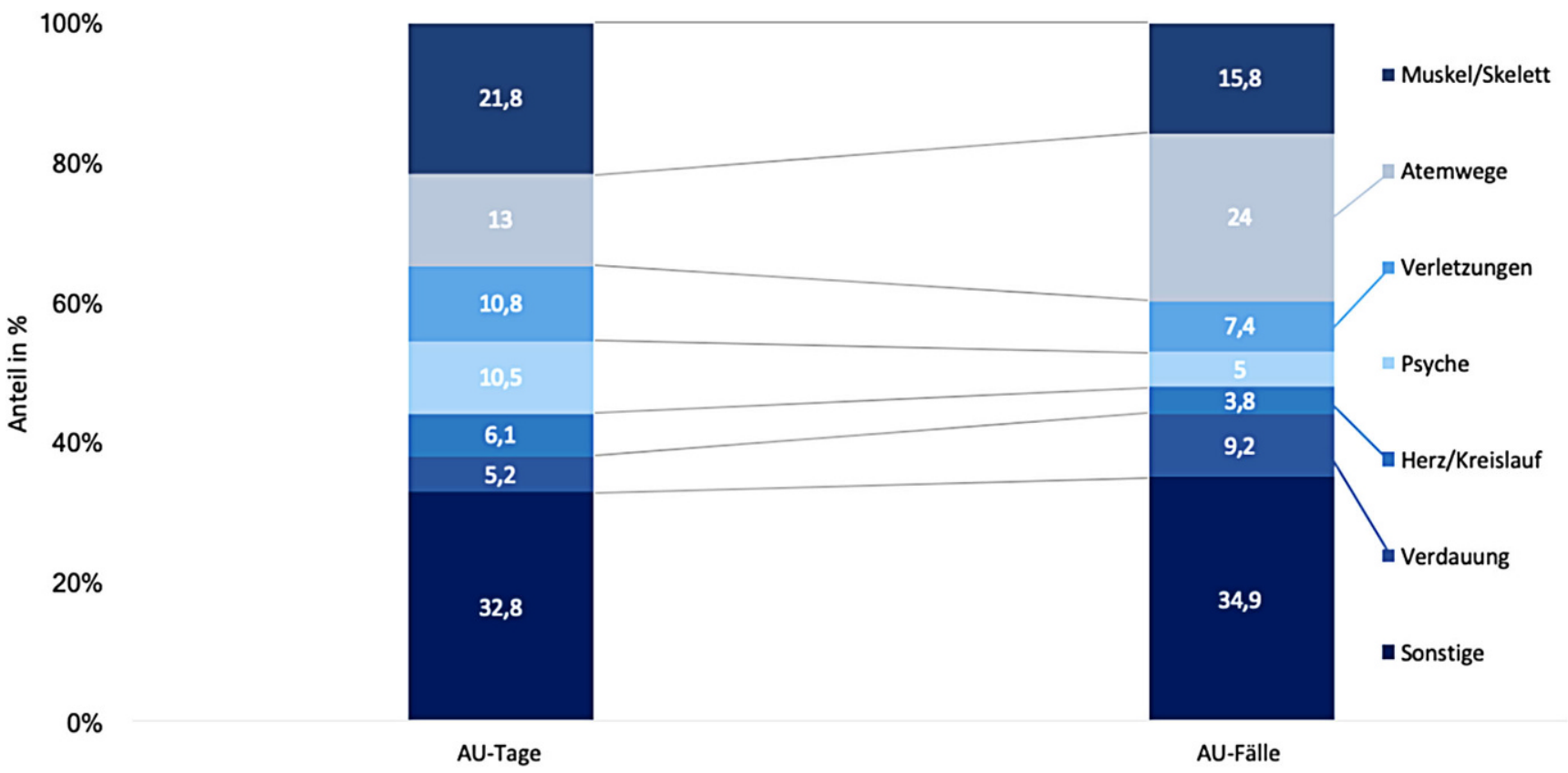

Abb. 1 Gründe für Arbeitsunfähigkeit ( $A U$ ) von AOK-Mitgliedern in 2015. (Quelle: eigene Darstellung, in Anlehnung an Badura et al. 2016)

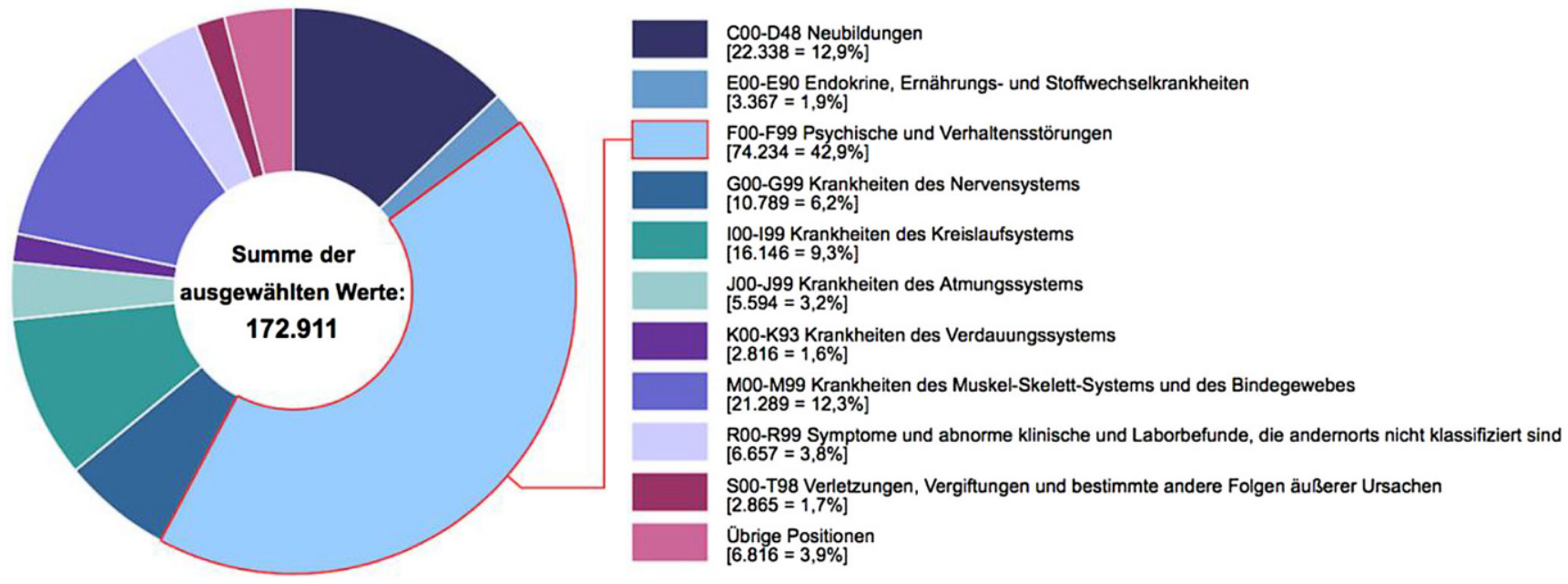

Abb. 2 Renteneintrittsgründe wegen verminderter Erwerbsfähigkeit in 2015. (Quelle: Gesundheitsberichterstattung des Bundes 2017)

Die Informationslage von Unternehmen über im Hause angefallene Fehlzeiten ergibt sich formal aus der ärztlichen Krankschreibung. Diese unterscheidet zwischen der Krankschreibung wegen Krankheit, wegen Krankheit eines Kindes, geplanter Krankheit etwa im Rahmen einer anstehenden Operation oder unattestiertem Fehlen wegen Krankheit bei Abwesenheitsdauer unter 3 Tagen (Dittmann et al. 2003). Die tatsächlichen Krankheitsursachen bleiben dem Unternehmen jedoch nicht zuletzt aus Datenschutzgründen (Bundesdatenschutzgesetz, §3 Abs. 9, §13 Abs. 2, §15 Abs. 5) meist verborgen und lassen deshalb keine expliziten Schlüsse über das Zustandekommen von Fehlzeiten zu. Grundsätzlich können Fehlzeiten jedoch als motivationsbe- dingt, krankheitsbedingt oder sonstige, etwa durch Fortbildungen bedingte, Ausfälle unterschieden werden (Gabler Wirtschaftslexikon 2019b). Aufgrund der dem Betrieb zur Verfügung stehenden Daten über Ausfälle und mit Blick auf die Fragestellung beschränkt sich diese Arbeit auf die Analyse von primärstatistisch verfügbaren Fehlzeiten.

Wichtige Herausforderungen, denen sich das Management von Fehlzeiten gegenübersieht, entstehen aus den gesellschaftlichen Entwicklungen. Hierzu gehören der demografische Wandel (Bundesanstalt für Arbeitsschutz und -medizin 2017) und der resultierende Fachkräftemangel. Durch höhere Arbeitsalter sinkt die physische Belastbarkeit des verfügbaren Personals, und krankheitsbedingte Ausfäl- 


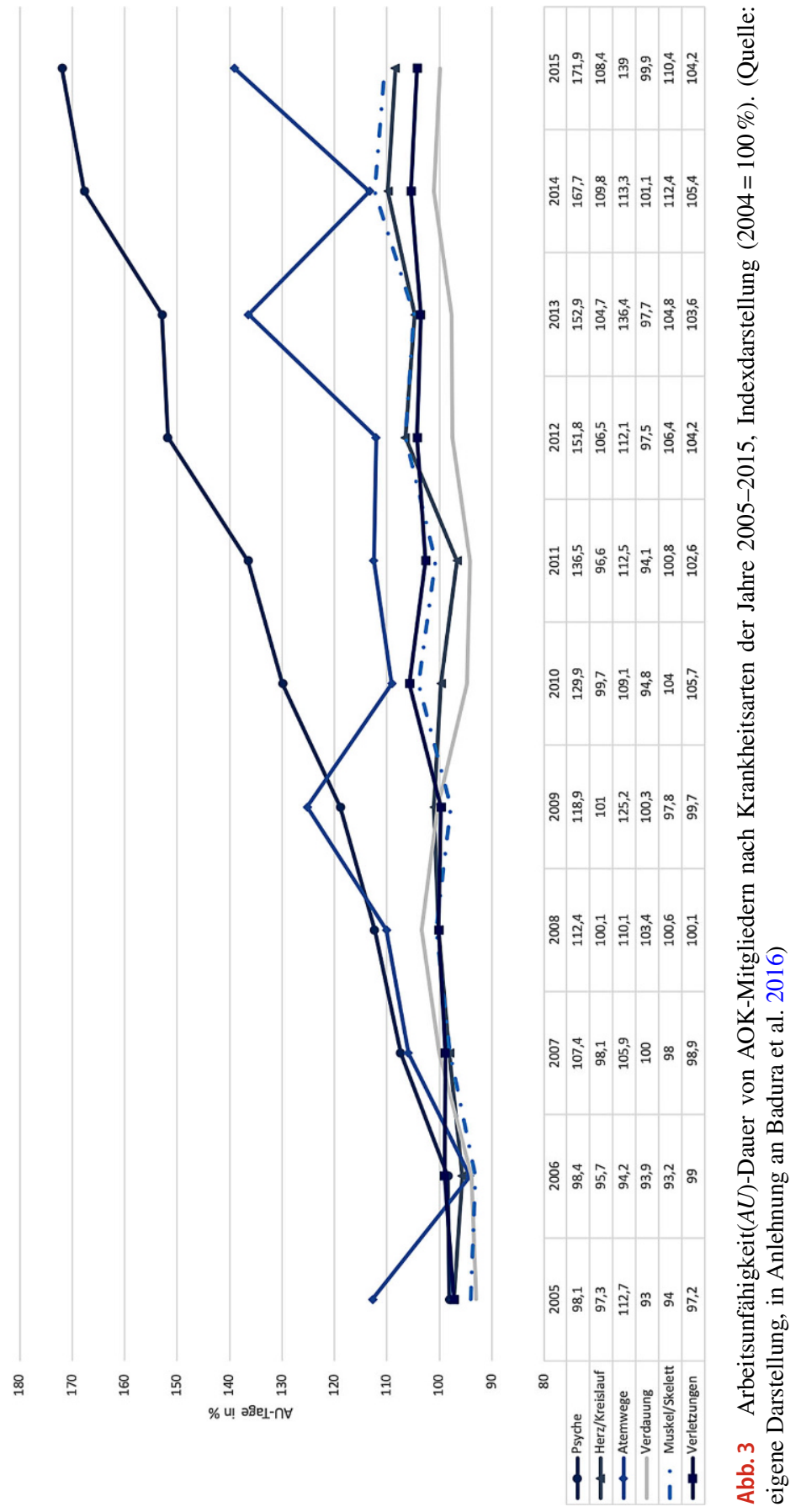


le belasten das bereits knappe Pflegepersonal zusätzlich. Laut Ergebnissen des branchenübergreifenden Fehlzeitenreports verursachen die im Alter zunehmenden Muskelund Skeletterkrankungen einen wesentlichen Anteil der registrierten Arbeitsunfähigkeiten (Abb. 1).

Zudem kann abnehmende Lern- und Weiterbildungsbereitschaft zusätzlich gesundheitsbeeinträchtigend wirken, etwa wenn durch sie Stress entsteht (Brandenburg und Nieder 2009). Die fortschreitende epidemiologische Transition (Omran 1971) führt zu einem veränderten Krankheitspanorama, in der chronisch-degenerative Krankheiten an Bedeutung gewinnen (Omran 2005). Neben diesen erlangten psychische Krankheiten in den vergangenen Jahren an Bedeutung (Murray und Lopez 1997; Nefiodow 2001). Unterstützt wird diese Aussage durch die Abb. 2 und 3, die u.a. den starken Anstieg von Arbeitsunfähigkeiten durch psychische Erkrankungen darstellt. Die häufiger werdende Diagnose psychischer Krankheiten wird mit gestiegener Sensibilität der Ärzte, erhöhten bzw. veränderten Arbeitsbelastungen oder zunehmender gesellschaftlicher Akzeptanz unterschiedlich begründet (Badura et al. 2016).

\section{Problemstellung und Zielsetzung}

Die formale Krankschreibung ist eine ärztliche Objektivierung des ansonsten subjektiv wahrgenommenen Gesundheitszustandes. Der tatsächliche Krankheits- bzw. Ausfallgrund bleibt dem Arbeitgeber verborgen, weshalb die Entwicklung von Maßnahmen zur Reduktion von Fehlzeiten im Rahmen des Managementzyklus erschwert wird. Auch motivationale Gründe für Ausfälle des Pflegepersonals, wie Überlastungen des Einzelnen durch Präsentismus oder zusätzliche Belastungen vieler durch den Absentismus einzelner Kollegen, stehen einem Unternehmen nicht unmittelbar Verfügung (Brandenburg und Nieder 2009). Aufgrund der fehlenden Informationen über Ausfallursachen wird die Entwicklung von Maßnahmen zum Management von Fehlzeiten erschwert.

Ziel dieser Studie ist es, die Grundlage für die Entwicklung wirksamer Handlungen zur Reduktion von Fehlzeiten des Pflegepersonals zu schaffen, indem ein systematischer Rahmen für die Analyse von Fehlzeiten entwickelt und angewendet wird. Die Voraussetzung für die Analyse von Fehlzeitendaten ist dabei das Verständnis der komplexen und dynamischen Beziehungen, die zur Einschränkung des Gesundheitszustandes und indes zu Produktivitätsverlusten führen können. Diese werden im Fehlzeitenmodell, das im Rahmen des methodischen Vorgehens vorgestellt wird, strukturiert und eingeordnet. Von einem maximalversorgenden Krankenhaus bereitgestellte Fehlzeitendaten des Pflegepersonals werden schließlich auf das Modell angewendet.

\section{Methodik}

\section{Analysevorgang}

Die Ursachen krankheitsbedingter Fehlzeiten von Pflegekräften sind als dynamisch, komplex und multikausal zu betrachten. Um diese Komplexität zu erfassen, wurde für einen systematischen Überblick über die verschiedenen Determinanten der menschlichen Arbeitsleistung auf Grundlage der Überlegungen von Berthel und Becker (2009) sowie Pfeiffer et al. (1988) ein auf den Pflegeberuf ausgerichtetes Modell entwickelt (Abb. 4). Dieses schlägt einen strukturellen Rahmen für die Methodik künftiger Fehlzeitenanalysen vor, indem Informationen über Ausfallzeiten systematisch festgelegten Fehlzeitenursachen zugeordnet werden. Aus dieser Zuordnung entsteht ein Profil, aus dem Ansätze zur Verringerung von Fehlzeiten abgeleitet werden können.

Krankheit verringert die Leistungsfähigkeit und verursacht im Fall von Arbeitsunfähigkeit den vollständigen Produktivitätsverlust einer Pflegekraft. An dieser Stelle entstehen dem Gesundheitsdienstleister krankheitsbedingte Fehlzeiten. Diese stellen Störgrößen im Leistungserstellungsprozess dar. Je genauer die Ursache der Störgröße bekannt ist, desto besser lässt sie sich einordnen, um auf sie reagieren zu können. Leistungsfähigkeit und die Darstellung ihrer Einflussgrößen sind deshalb zentrale Bestandteile einer Fehlzeitenanalyse und finden sich als solche im entwickelten Fehlzeitenmodell wieder. Die Verfehlung eines der Elemente des Modells muss als notwendige Bedingung für die Entstehung von Fehlzeiten, Erkrankungen oder Motivationsverlust gesehen werden. Dabei ist die einzelne Verfehlung aber nicht zwingenderweise eine hinreichende Begründung. Die Ursachen der im Modell beschriebenen Leistungseinbußen sind multikausal und interdependent. Eine Einzelbetrachtung greift aufgrund der komplexen Struktur der Elemente und ihrer Dynamik zu kurz. Dementsprechend muss die Möglichkeit einer Kausalbeziehung zwischen einem Element und entstandenen Leistungsverlusten berücksichtigt werden, sie ist jedoch nicht pauschal zu unterstellen. Andere Ursachen des Leistungsverlustes sind immer zu prüfen und ihre Relationen untereinander zu betrachten. Aufgrund des ganzheitlichen Ansatzes zur Erklärung von Leistungsverlusten kann den vorgestellten Elementen bei Berücksichtigung ihrer Relationen Kausalität unterstellt werden, sodass eine Bezeichnung als Kausalmodell Rechtfertigung findet.

Das entwickelte Modell stellt folgende Beziehungen dar: Intra- und extrapersonelle Determinanten (nach Pfeiffer et al. 1998) bedingen die mögliche Dauer und Intensität der menschlichen Arbeit. Durch den Einsatz von Arbeitskraft über einen bestimmten Zeitraum hinweg ergibt sich schließlich die Leistung. Diese wird entlohnt. Das Ergebnis des dem Leistungsdeterminantenkonzept (Berthel und Be- 


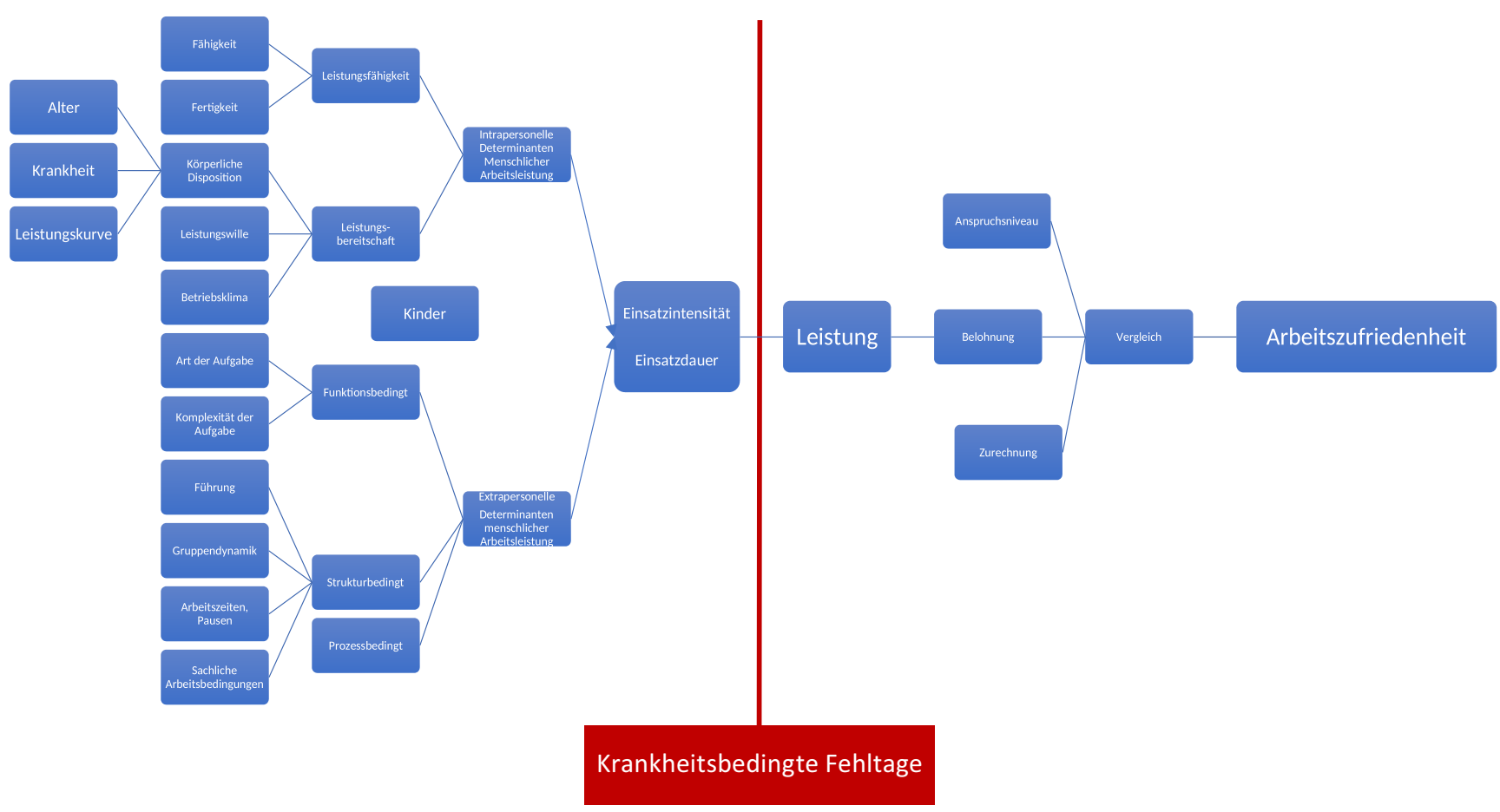

Abb. 4 Kausalmodell. (Quelle: eigene Darstellung)

cker 2009) folgenden Vergleichs der Entlohnung, mit der die erbrachte Leistung monetär bewertet wird, ergibt sich aus dem individuellen Anspruchsniveau sowie der Zurechnung. Diese bezieht sich auf die Rückführung von Erfolgen oder Misserfolgen auf interne und beeinflussbare oder externe nichtbeeinflussbare Faktoren. Das subjektive Ergebnis des Vergleichs schlägt sich in der Arbeitszufriedenheit der Pflegekräfte nieder. Diese wiederum beeinflusst maßgeblich die Ausprägung der intra- und extrapersonellen Determinanten einerseits und mithin die mögliche Arbeitsleistung durch Einsatzintensität und -dauer andererseits. Trotz der scheinbaren Nähe zu der intrapersonellen Determinante der Leistungsbereitschaft steht die Arbeitszufriedenheit als eigenes Element, um mit dem ihr zugeordneten Vergleich explizit auf die Motivation des Personals eingehen zu können.

Um seine Geltung zu beweisen, muss das Modell Gesetzmäßigkeiten der Fehlzeitenanalyse wiedergeben und nichtabsehbare Ergebnisse einordnen können, als nachträgliche Erweiterung oder in den bestehenden Rahmen. Hierfür muss besondere Aufmerksamkeit auf die Determinanten der menschlichen Arbeitskraft gelegt werden, welche die Pflegekraft umfassend charakterisieren und den im Modell dargestellten Kreislauf maßgeblich beeinflussen.

Intrapersonelle Determinanten liegen in der Pflegekraft selbst begründet und weisen große Überschneidung mit den persönlichen Faktoren nach Brandenburg und Nieder (2009) auf. Fähigkeiten und Fertigkeiten ergeben die individuelle Leistungsfähigkeit, also die Eignung, eine Arbeits- leistung in vorgegebener Zeit und Güte zu erbringen. Unterscheiden sich angeborene und erworbene Anlagen erheblich von der Aufgabenstellung, können krankheitsbedingte Fehlzeiten durch physische oder psychische Belastungen und somit Leistungsverluste entstehen. Neben der Leistungsfähigkeit bedingt die Leistungsbereitschaft maßgeblich die Einsatzintensität und -dauer. Der Übergang der Modellgröße des Betriebsklimas zu den strukturbedingten extrapersonellen Determinanten ist fließend. Nach dem Leistungswillen der Pflegekraft, der unterschiedliche Theorien zu Menschenbildern und Motivation aufgreift, ist insbesondere die körperliche Disposition hervorzuheben. Tagesrhythmik, physiologische Leistungskurve sowie schlecht verheilte oder chronische Erkrankungen können grundsätzlich die Leistungsbereitschaft, die Einsatzintensität und -zeit verändern. Trifft verschlechterte körperliche Disposition auf ungünstige extrapersonelle Determinanten, können gesundheitseinschränkende Effekte vermehrt auftreten.

Die Modellelemente Arbeitsbedingungen und teilweise auch betriebliche Rahmenbedingungen können in Bezug auf Brandenburg und Nieder (2009) den extrapersonellen Determinanten menschlicher Arbeitskraft zugeordnet werden. Die Ausführungen zur Entstehung von Fehlzeiten in früheren Abschnitten sind besonders dem Bereich der funktionsbedingten extrapersonellen Determinanten zuzuweisen. Funktionsbedingte Einflussfaktoren können psychischer und physischer Art sein; sie entstehen durch die Art und Komplexität der jeweiligen Aufgabe. Die Komplexität wird bedingt durch die Zahl der Elemente und ihrer 
Relationen. Je höher die Komplexität der Aufgabe, desto höher sind die Anforderungen, die an den die Aufgabe Lösenden gestellt werden. Die Anforderungsarten können mit der Art der Aufgabe beschrieben werden. Diese können an Ausbildung oder Erfahrung gestellt werden; andere Anforderungsarten sind beispielsweise als muskulär oder geistig einzuordnen. Passen personelle Faktoren nicht zu den funktionsbedingten Anforderungen, kann die resultierende Über- oder Unterforderung zu Ausfällen unterschiedlicher Art führen.

Im Rahmen der strukturbedingten extrapersonellen Determinanten können durch den Führungsstil, die Gruppendynamik, Arbeitszeiten oder durch sachliche Arbeitsbedingungen Leistungsminderungen entstehen. Strukturbedingte Determinanten umfassen die Arbeitsumgebung, mit der eine Pflegekraft konfrontiert wird. Zur Führung und den mit ihr verbundenen Führungs- und Motivationstheorien sowie Persönlichkeitstypologien sei an dieser Stelle auf diverse Ansätze wie die Prinzipal-Agent- oder „Stewardship“-Theorie, die Maslow-Bedürfnispyramide oder Persönlichkeitstypisierungen verwiesen. Das Verhalten der Führungskraft bei der Verteilung und Leitung der Arbeitsaufgaben sowie der Gestaltung der Beziehungen innerhalb der Arbeitsgruppe beeinflusst entscheidend die Leistungsbereitschaft und -intensität der Geführten. Gruppendynamik entsteht aus der Interaktion verschiedener Charaktere. Die Phasen der Gruppenarbeit werden u.a. von umfassend Rieckmann (Rieckmann 2005) beschrieben. Eine positiv ausgeprägte Dynamik wirkt sich steigernd auf die Dauer und Intensität des Einsatzes aus, jedoch ist auch das Gegenteil möglich. Neben den sachlichen Arbeitsbedingungen sind insbesondere die Arbeitszeiten des Pflegepersonals zu berücksichtigen. Diese umfassen den Arbeitszeitraum, Schichtdienste, Pausen und Über- oder Mehrstunden. Das dritte Element der extrapersonellen Determinanten sind die prozessbedingten. Es umfasst die von der Prozessstruktur vorgegebene und deshalb nicht individuell beeinflussbare Arbeitsmethodik, mit der Aufgaben verrichtet werden, und impliziert aus dieser entstehende Belastungen.

Eine weitere wichtige Rolle für die Analyse von Fehlzeiten im Pflegeberuf spielt die Anzahl der Kinder. Dieses Element des Kausalmodells weist weite Überschneidungen mit den anderen Faktoren des Modells auf und steht zur Abgrenzung für sich. Kinder als möglicherweise sinngebender Lebensinhalt können sich auf verschiedene Arten auf die Arbeitsleistung auswirken. Einerseits können sie als Ansporn für einen höheren Lebensstandard oder Sicherheit stehen. Die zeitintensive Erziehung kann sich jedoch auch in Belastungen niederschlagen, durch welche die Produktivität beeinträchtigt wird. So kann die Krankschreibung im Betrieb auch dann erfolgen, wenn der Erziehungsberechtigte gesund ist, das Kind jedoch krank. Schlafmangel oder das Tragen der Kinder können zudem langfristig Auswirkungen auf den physischen oder psychischen Gesundheitszustand zeigen.

Störgrößen, die sich aus einer Strukturanalyse ergeben, können in die Beziehungen der Elemente des Modells eingeordnet werden. Die Ausprägung einer oder mehrerer Störgrößen auf eines oder mehrere betroffene Elemente entscheidet über die Entstehung von Fehlzeiten. Der im Kausalmodell beschriebene Kreislauf aus Leistungsabgabe, Entlohnung und Zufriedenheit, durch die wiederum die leistungsbedingenden Faktoren beeinflusst werden, dient als Struktur zur Einordnung der folgend dargestellten Analyseergebnisse.

\section{Material}

Der Arbeit liegen primärstatistisch erhobene Daten eines maximalversorgenden Krankenhauses mit ca. 900 Betten zugrunde. Bereitgestellt wurden 73.996 im SAP-System registrierte Ausfälle von rund 1500 Pflegekräften in den Jahren 2015 und 2016. Zur Gewährleistung des Datenschutzes wurden den Personen zufällige 6-stellige Identifikationsnummern zugewiesen. Bekannt und zuordenbar waren der jeweilige Ausfallgrund laut Krankschreibung bzw. das unattestierte Fehlen weniger als 3 Tage, die Station der Pflegekraft, Alter, Geschlecht, Zahl der Kinder, Beginn des Beschäftigungsverhältnisses, Stellenumfang sowie die berufliche Qualifikation. Weitere Informationen wurden aus sekundärstatistisch verfügbaren Mitarbeiterbefragungen der Jahre 2012 und 2014 gewonnen.

Um Zusammenhänge, auffällige Strukturen oder Regelmäßigkeiten der Fehlzeiteninformationen herauszuarbeiten, wurden univariate deskriptive Methoden zu Verdichtung und Darstellung der Daten sowie explorative statistische Methoden verwendet. Für die Beschreibung der Ergebnisse auf Grundlage der statistischen Verfahren wurden u. a. Boxplots, Dichtekurven, Gini-Koeffizienten sowie Kennzahlen zu Lage- und Streuungsmaßen eingesetzt. Die methodische Umsetzung erfolgte mit den Programmen Microsoft Excel und R Studio.

\section{Ergebnisse}

Der den Modelldeterminanten unterstellte Kausalzusammenhang ermöglichte die Analyse im Rahmen der Modellüberlegungen. Folgende Darstellung wesentlicher Ergebnisse bezieht sich auf das Jahr 2016, in dem 1480 Pflegekräfte beschäftigt waren, bei einem Durchschnittsalter von 39 Jahren. Rund $16 \%$ der Pflegekräfte waren männlich. In einer rechtsschiefen Verteilung (Gabler Wirtschaftslexikon 2019b) betrugen die mittleren Fehlstunden $157(\mathrm{~d}=273,85)$ Stunden pro Kopf im Jahr, wobei auf 529 Pflegekräfte überhaupt keine Fehlstunden entfielen. Der 
Abb. 5 Verteilung jährlicher Fehlstunden pro Kopf in 2015 und 2016. (Quelle: eigene Darstellung)
Abb. 6 Durchschnittliche Fehlstunden im Jahr nach Geschlecht und Altersgruppe. (Quelle: eigene Abbildung)
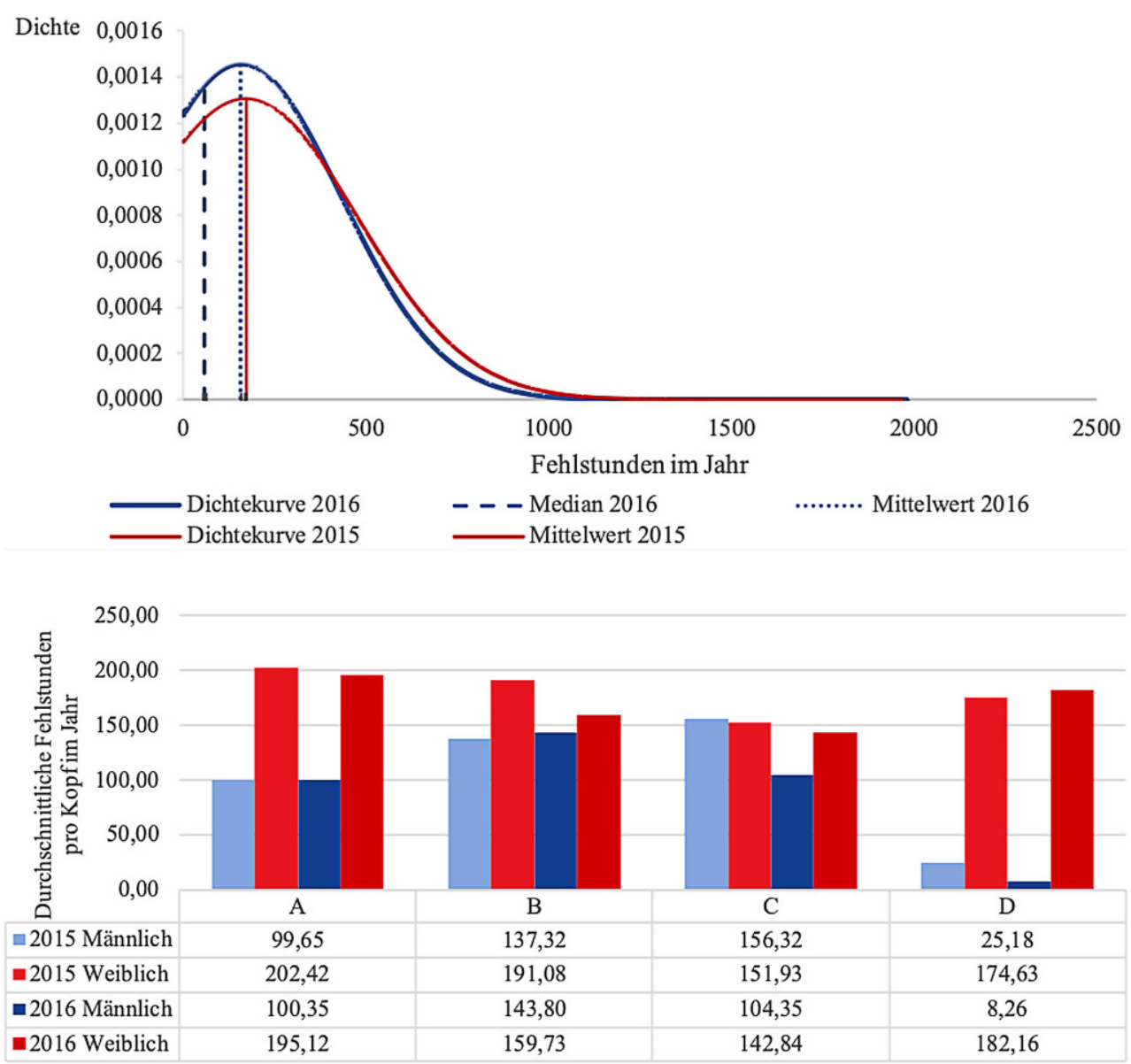

Gini-Koeffizient von 0,72 weist entsprechend auf eine hohe Konzentration der Fehlzeiten auf einen kleinen Teil der betrachteten Belegschaft hin (Abb. 5).

Im Rahmen der intrapersonellen Determinanten liegt zunächst die Betrachtung der Unterschiede zwischen Männern und Frauen nahe. Um Vergleichbarkeit zu schaffen, dürfen dabei jedoch schwangerschaftsbedingte Fehlzeiten nicht berücksichtigt werden. So zeigt sich, dass männliche Pflegekräfte unter 39 Jahren im Schnitt sowohl häufiger fehlten als Frauen gleichen Alters als auch häufiger als jene Männer mit mehr als 40 Jahren. Für weibliche Pflegekräfte ist bei steigendem Alter eine deutliche Zunahme der durchschnittlichen krankheitsbedingten Fehlstunden im Jahr zu erkennen; so fehlten Pflegerinnen unter 29 Jahren etwa $89 \mathrm{~h}$ im Jahr, während jene zwischen 50 und 69 Jahren im Mittel rund 182 Fehlstunden im Jahr verursachten (Abb. 6).

Häufungen von Ausfällen zu bestimmten Wochentagen können nicht erkannt werden, sodass die Arbeitszeiten während der Arbeitswoche keinen Einfluss auf die Leistungskurve zu haben scheinen. Bei quartalsweiser Betrachtung des Anfalls von Fehlstunden lässt sich jedoch beobachten, dass im Sommerhalbjahr weniger Ausfälle verzeichnet werden.
Schwangerschaftsbedingte Ausfälle stellen erwartungsgemäß einen Fehlzeitentreiber dar, auf den rund $23 \%$ der angefallenen Fehlzeiten zurückgeführt werden können. Bereits der durchschnittliche schwangerschaftsbedingte Arbeitsausfall mit ca. $725 \mathrm{~h}$ ist hinsichtlich des Zeitraums als statistischer Ausreißer einzuordnen (Abb. 7).

Besonders für die Analyse funktionsbedingter und strukturbedingter Determinanten (Abb. 4), wie auch von Belohnung und Zurechnung, war neben den Fehlzeitendaten die Mitarbeiterbefragung $(n=269)$ aus 2014 nützlich. Nach dieser gaben 43,5\% der befragten Pflegekräfte an, ihre Arbeit als Mittel zum Lebensunterhalt zu sehen; die wichtigen Dinge würden in der Freizeit geschehen. Zudem scheinen sich rund $40 \%$ der befragten Pflegekräfte nicht mit ihrem Arbeitgeber identifizieren zu können. Ähnliche, Unzufriedenheit implizierende Ergebnisse gleicher Höhe finden sich bei den Fragen zur Zufriedenheit mit der Bezahlung, mit der Führung, der Informationsweitergabe sowie den Arbeitszeiten. Eine Betrachtung der Ausfallstunden der jeweiligen Organisationseinheiten und der ihr zugeordneten Pflegekräfte zeigt stark schwankende jährliche Mittelwerte, zwischen 90 und $486 \mathrm{~h}$. 


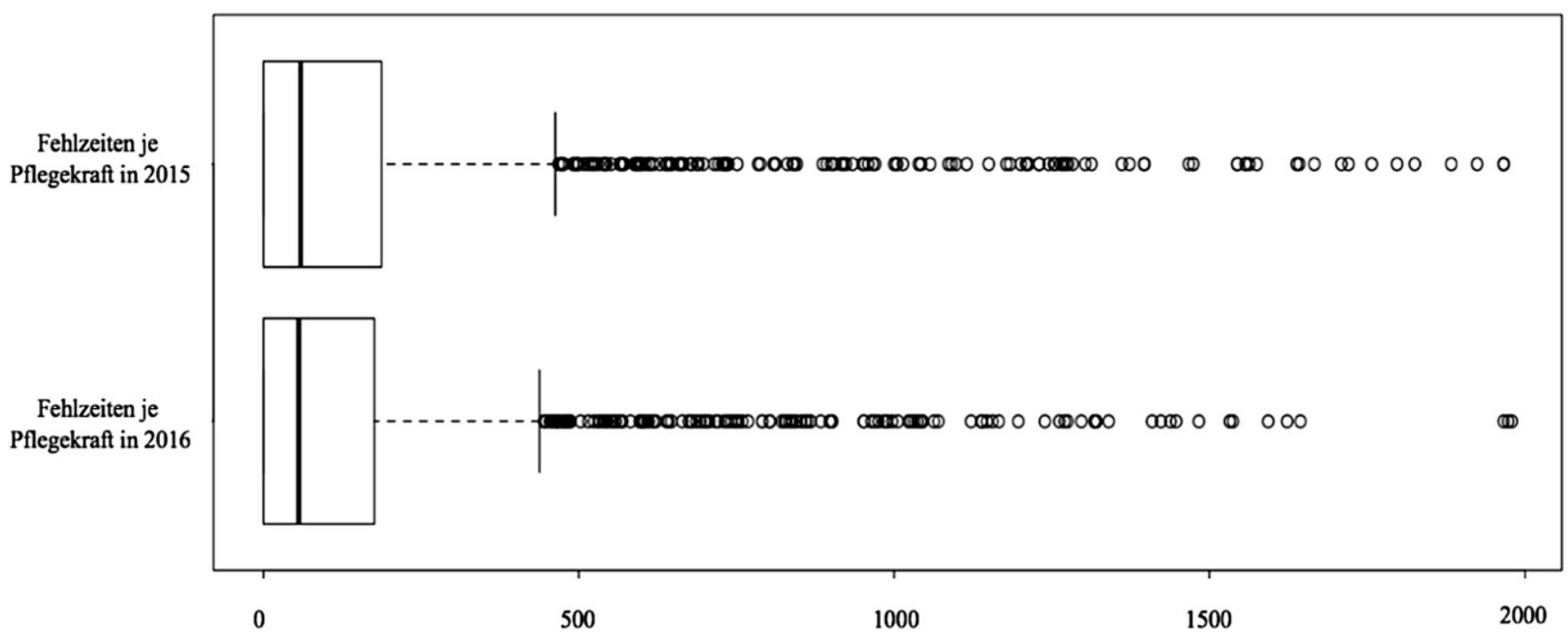

Abb. 7 Boxplot für krankheitsbedingte Fehlzeiten in 2015 und 2016. (Quelle: eigene Darstellung)

\section{Diskussion}

Die von einem maximalversorgenden Krankenhaus im Rahmen dieser Studie bereitgestellten Fehlzeitendaten wurden im Rahmen des entwickelten Kausalmodells entsprechend der Zielsetzung dieser Arbeit geordnet und systematisch analysiert. Während die intrapersonellen Determinanten (Abb. 4) einen guten Überblick der Fehlzeitenstruktur des Pflegepersonals ermöglichen, sind Ergebnisse zu extrapersonellen Determinanten (Abb. 4) weniger deutlich und lassen Interpretationsräume. Ein knappes Viertel der in 2016 in dem betrachteten maximalversorgenden Krankenhaus angefallenen Fehlzeiten, und indes vermutlich auch bis zu einem gewissen Grade deren hohe Konzentration auf einen Teil der Belegschaft, lassen sich mit Schwangerschaften begründen. Eindeutige Kenntnis, wie hoch und aus welcher Ursache die krankheitsbedingten Fehlzeiten, abgesehen von den schwangerschaftsbedingten, über dem anzunehmenden Fehlzeitensockel (Brandenburg und Nieder 2009) liegen, vermag die Arbeit, nicht zuletzt aus Datenschutzgründen, nicht zu liefern.

Dringend einzugehen ist auf ein mögliches Motivationsproblem der Belegschaft, das durch die letzte Mitarbeiterbefragung aufgeworfen wurde. Sollte dieses bei einigen Pflegekräften ein grundsätzliches Problem darstellen, würde das allgemein angenommene Menschenbild der Pflegekraft als intrinsisch orientierte Helferpersönlichkeit (Schmidbauer 1978) geprüft werden müssen. Ist dies nicht der Fall, könnte die Unzufriedenheit das Resultat extrapersoneller Einflüsse sein. Hierzu würden beispielsweise im Rahmen der strukturbedingten Determinanten der Führungsstil des Vorgesetzten, die Gruppendynamik oder die sachlichen Arbeitsbedingungen der Pflegekraft zählen. Im weiteren Vorgehen des Arbeitgebers muss hier dringend Problemkennt- nis erlangt werden. Die Möglichkeit von Absentismus, der von einem Motivationsproblem impliziert würde, könnte zunächst mit einer nach 4 Jahren überfälligen neuen Mitarbeiterbefragung überblickt werden. Zudem könnte mithilfe von regelmäßigen Mitarbeitergesprächen, also Feedback-Schleifen, die Arbeitsumgebung auf den einzelnen Stationen besser eingeschätzt werden. Derartige Gespräche zwischen Vorgesetzten und Mitarbeitern werden hinsichtlich ihrer Wirkung in der Literatur kontrovers diskutiert (Ulich und Wülser 2012; Pfaff et al. 2002; Backes-Gellner et al. 2001). Der Kontakt zwischen Vorgesetzten und Pflegekräften bedarf in diesem Fall jedoch offensichtlich einer Verbesserung. Durch verstärkten persönlichen Bezug zwischen Pflegekräften und Arbeitgeber könnte eine etwaige Defektion des Arbeitgebers durch die Pflegekraft, wie sie in der Mitarbeiterbefragung aufgeworfen wird, von vornherein vermieden werden. Der Fokus solcher Audits sollte zudem nicht nur auf der Krankenpflege liegen, sondern auch auf dem Führungspersonal der Organisationseinheiten.

Darüber hinaus könnten die stark schwankenden durchschnittlichen Fehlzeiten der unterschiedlichen Stationen geglättet werden, indem eine homogenere Personalstruktur an den Organisationseinheiten umgesetzt wird. Im Rahmen einer Umstrukturierung, beispielsweise an den Stationen, könnte die Erhöhung des bisher geringen Anteils von $16 \%$ der männlichen Pflegekräfte an der Belegschaft angestrebt werden. Auch die Altersstruktur an den jeweiligen Organisationseinheiten könnte angeglichen werden. Somit könnten etwa schwere körperliche Arbeiten besser verteilt und Fehlzeiten leichter gesteuert werden. Zudem ließen sich Häufungen schwangerschaftsbedingter Ausfälle an Stationen vermeiden.

Die beschriebenen Möglichkeiten zur Vermeidung der Häufung von krankheitsbedingten Ausfällen an bestimmten 
Stationen und die daraus folgende zusätzliche Arbeitsbelastung für das gesunde Personal ist gleichzeitig eine Möglichkeit, die Gesundheit derjenigen Pflegekräfte mit besonders wenigen Ausfällen zu schützen. Sie kompensieren den Arbeitsausfall anderer Kollegen, deshalb muss es Ziel des Betriebs sein, einerseits ihre Arbeitskraft zu erhalten. Andererseits muss ein Abwandern dieser Personengruppe unbedingt verhindert werden.

Die Maßnahmen zur Optimierung der Personalstruktur und die damit einhergehende Mitarbeiterakquise, der Erhalt der Arbeitskraft sowie Einsatz und Führung der Pflegekräfte finden im Rahmen eines sehr angespannten Arbeitsmarktes statt. Die dargestellten Maßnahmen müssen deshalb Teil einer Strategie zum Arbeitsmarktauftritt sein und in dieser als Wettbewerbsvorteile entwickelt werden.

Eine wichtige Limitation dieser Arbeit ist die verarbeitete Datengrundlage. Um allen Dimensionen des erarbeiteten Modells entsprechend ein Bild über Leistungsverluste des Pflegepersonals zu erlangen, ist großer Bedarf an Daten und Informationen notwendig, der von den verfügbaren Fehlzeitendaten nicht vollends gedeckt werden konnte. Weiterer Forschung und vertiefter Informationslage steht, wie bereits in dieser Arbeit, der Datenschutz als wesentliche Hürde entgegen.

Das entwickelte Fehlzeitenmodell erwies sich in der vorgenommenen Fehlzeitenanalyse als sehr hilfreiche gedankliche Stütze. Jedoch ergibt sich aus der modellierten Denkweise die Gefahr, dass Entwicklungen oder Ergebnisse der Analyse fälschlicherweise in das Modell eingeordnet werden, obwohl es für diese einer Erweiterung des Modellrahmens bedurft hätte. Die mitunter nichteindeutige Trennung der Elemente des Kausalmodells führt zudem dazu, dass Daten oder Relationen falsch eingeordnet werden könnten.

\section{Schlussfolgerung}

Die vorliegende Arbeit zeigt, dass eine systematische Analyse krankheitsbedingter Fehlzeiten mithilfe des oben skizzierten Fehlzeitenmodells möglich ist. Neben Daten aus der ärztlichen Krankschreibung konnten alle zusätzlichen, zum Pflegepersonal des betrachteten Krankenhauses verfügbaren Informationen über Abwesenheiten eingeordnet und in ursächlichen Bezug gesetzt werden. Der modellierte Denkrahmen kann von der Krankenhausleitung für künftige Fehlzeitenanalysen verwendet werden und liefert so Gesundheitsbetrieben ein besseres Verständnis zur Einschätzung von krankheitsbedingten Produktivitätseinbußen ihres Pflegepersonals. Maßnahmen wie die Homogenisierung der Personalstruktur oder die Herstellung eines verbesserten persönlichen Kontakts durch Mitarbeitergespräche zur Verringerung von Fehlzeiten lassen sich ableiten. Ihre Umsetzung, z.B. im Rahmen des Qualitätsmanagements, er- fordert jedoch eine strategische Managemententscheidung, Priorität innerhalb des Führungshandelns und ausreichende finanzielle Mittel, um eine Verbesserung der Gesundheit der Mitarbeiter und damit eine nachhaltige Reduktion der Fehlzeiten zu ermöglichen.

Funding Open Access funding provided by Projekt DEAL.

Open Access Dieser Artikel wird unter der Creative Commons Namensnennung 4.0 International Lizenz veröffentlicht, welche die Nutzung, Vervielfältigung, Bearbeitung, Verbreitung und Wiedergabe in jeglichem Medium und Format erlaubt, sofern Sie den/die ursprünglichen Autor(en) und die Quelle ordnungsgemäß nennen, einen Link zur Creative Commons Lizenz beifügen und angeben, ob Änderungen vorgenommen wurden.

Die in diesem Artikel enthaltenen Bilder und sonstiges Drittmaterial unterliegen ebenfalls der genannten Creative Commons Lizenz, sofern sich aus der Abbildungslegende nichts anderes ergibt. Sofern das betreffende Material nicht unter der genannten Creative Commons Lizenz steht und die betreffende Handlung nicht nach gesetzlichen Vorschriften erlaubt ist, ist für die oben aufgeführten Weiterverwendungen des Materials die Einwilligung des jeweiligen Rechteinhabers einzuholen.

Weitere Details zur Lizenz entnehmen Sie bitte der Lizenzinformation auf http://creativecommons.org/licenses/by/4.0/deed.de.

\section{Literatur}

Backes-Gellner U, Schorn R, Krings A (2001) Ursachen und Abbau von Fehlzeiten: Analysen auf Basis einer mehrjährigen Betriebsfallstudie. Z Betriebswirtsch 1/2001:105-117

Badura B, Ducki A, Schröder H, Klose J, Meyer M (Hrsg) (2016) Fehlzeiten-Report 2016. Springer, Berlin

Berthel J, Becker F (2009) Personal-Management: Grundzüge für Konzeptionen betrieblicher Personalarbeit. Schäffer-Poeschel, Stuttgart

Brandenburg U, Nieder P (2009) Betriebliches Fehlzeiten-Management. 2. Aufl. Gabler, Wiesbaden

Bundesanstalt für Arbeitsschutz und Arbeitsmedizin (2017) Arbeit in der Pflege - Arbeit am Limit? Arbeitsbedingungen in der Pflegebranche. www.baua.de/dok/6505548. Stand 29.11.2017

Deutscher Berufsverband für Pflegeberufe (1992) Berufsordnung für Pflegeberufe. DBfK-Verlag, Eschborn

Dittmann A, Müller H-J, Schröder-Kamprad T (2003) Gesundheit als Wettbewerbsfaktor Bd. 1. Landesunfallkasse Freie und Hansestadt Hamburg, Hamburg

Duppel S (2005) Nähe und Distanz als gesellschaftliche Grundlegung in der ambulanten Pflege. Schlütersche, Bremen

Fleßa S (2012) Internationales Gesundheitsmanagement. Oldenbourg, München

Fleßa S (2013) Grundzüge der Krankenhausbetriebslehre. Oldenbourg, München

Gabler Wirtschaftslexikon (2019a) https://wirtschaftslexikon.gabler. de/definition/fehlzeiten-32768/version-256304. Zugegriffen: 15. Jan. 2019

Gabler Wirtschaftslexikon (2019b) https://wirtschaftslexikon.gabler. de/definition/schiefe-45767/version-269055. Zugegriffen: 15. Apr. 2019

Gesundheitsberichterstattung des Bundes (2017) Zugänge in der GRV wegen verminderter Erwerbsfähigkeit im Jahr 2015. http://www. gbe-bund.de. Stand 29.11.2017

Glaser J, Höge T (2005) Spezifische Belastungen und Anforderungen personenbezogener Krankenhausarbeit. In: Badura B (Hrsg) Fehlzeitenreport 2004. Springer, Berlin, S 51-64 
Herrmannsdorfer U (2000) Wege zur Qualität: Die Befreiung Schöpferischer Kräfte (https://www.sozialimpulse.de/fileadmin/pdf/ Qualitaet.pdf)

Murray C, Lopez A (1997) Alternative projections of mortality and disability by cause 1990-2020: Global Burden of Disease Study. Lancet 349:1498-1504

Nefiodow L (2001) Der sechste Kondratieff, 5. Aufl. Rhein-Sieg Verlag, Sankt Augustin

Omran A (1971) The epidemiologic transition. A theory of epidemiology of population change. Milbank Meml Fund Q 49:509-538

Omran A (2005) The epidemiologic transition: a theory of the epidemiology of population change. Milbank Q 83:4

Pfaff H, Kaiser C, Krause H (2002) Krankenrückkehrgespräche: Zur Ambivalenz einer Sozialtechnologie. Gutachten für die Expertenkommission „Betriebliche Gesundheitspolitik“. Bertelsmann Stiftung, Hans-Böckler-Stiftung, Köln
Pfeiffer W, Dörrie U, Stoll E (1988) Menschliche Arbeit in der industriellen Produktion. Vandenhoeck \& Ruprecht, Göttingen

Rieckmann H (2005) Management und Führen am Rande des 3. Jahrtausends. Lang, Frankfurt a.M.

Schmidbauer W (1978) Die hilflosen Helfer: Über die seelische Problematik der helfenden Berufe, 32. Aufl. Rowohlt, Reinbek

Ulich E, Wülser M (2012) Gesundheitsmanagement im Unternehmen. Gabler, Wiesbaden

World Health Organisation (1948) Verfassung der Weltgesundheitsorganisation. WHO, Genf. https://www.admin.ch/ch/d/sr/c0_810_1. html. Zugegriffen: 29.11.2017

Hinweis des Verlags Der Verlag bleibt in Hinblick auf geografische Zuordnungen und Gebietsbezeichnungen in veröffentlichten Karten und Institutsadressen neutral. 